\title{
Chebyshev-Type Integration Rules of Minimum Norm
}

\author{
By Philip Rabinowitz and Nira Richter
}

\begin{abstract}
Equal-weight integration rules are studied in the context of certain families of Hilbert spaces of analytic functions defined in a family of confocal ellipses containing the interval of integration. Rules which minimize the norm of the error functional in these spaces are shown to exist and several such rules are tabulated. Asymptotic properties of these rules are studied for ellipses shrinking to the integration interval and for ellipses expanding to cover the entire plane. In the latter case, an algebraic formulation for these asymptotic rules is given and it is shown that they agree with the classical Chebyshev integration rules whenever such rules exist.
\end{abstract}

1. Introduction. The classical Chebyshev integration problem is to find distinct real abscissae $x_{i}, i=1, \cdots, n$, and a weight $w$ such that, in the formula

$$
I(f) \equiv \int_{-1}^{1} f(x) d x=w \sum_{i=1}^{n} f\left(x_{i}\right)+E_{n}(f) \equiv R_{n}(f)+E_{n}(f),
$$

the error term $E_{n}(f)$ vanishes for all polynomials of degree $\leqq n$. It turns out that this problem only has a solution for $n=1(1) 7$ and $n=9$, and that in these cases $w=2 / n$. For all other values of $n$, some of the abscissae, which are the roots of a certain polynomial, turn out to be complex. In the sequel, we shall call any integration rule of the form

$$
R_{n}(f)=w \sum_{i=1}^{n} f\left(x_{i}\right), \quad-1 \leqq x_{1}<x_{2}<\cdots<x_{n} \leqq 1,
$$

a Chebyshev-type integration rule (CIR), while a rule of the same form in which we permit equality among abscissae will be called a generalized Chebyshev-type integration rule (GCIR). These latter come up as limiting forms of sequences of CIR's in various contexts.

In addition to the classical requirements on integration rules that they be exact for polynomials up to a specific degree, other criteria for integration rules have been proposed in recent years, among these being the requirement that they minimize the norm of the error functional $E_{n} f$ in a certain space. Rules with such a minimizing property are called optimal rules or minimal rules and have been discussed by various authors [1], [5], [7], [8]. To the authors' knowledge, the only work on CIR's with such minimal properties is that by Barnhill et al. [2] who computed CIR's which minimize the function

$$
\sum_{i=0}^{k}\left[E_{n} f\left(x^{i}\right)\right]^{2},
$$

Received November 14, 1969.

AMS 1969 subject classifications. Primary 6555.

Key words and phrases. Chebyshev integration rules, minimum norm rules, norm of error functional, Hilbert space, analytic functions, asymptotic integration rules, equal-weight integration rules. 
where $n \leqq k \leqq \infty$. For $k=n$, these rules turned out to be the classical rules, when such rules existed. In the other cases, the rules turned out to be GCIR's.

In the present work, we shall be concerned with CIR's which minimize the norm of $E_{n}(f)$ in two families of Hilbert spaces. Each family is a one-parameter family of Hilbert spaces of functions analytic in $\varepsilon_{\rho}, \rho>1$, where $\varepsilon_{\rho}$ is the ellipse in the complex plane with foci at $( \pm 1,0)$ and semimajor axis $a=\frac{1}{2}(\sqrt{ } \rho+1 / \sqrt{ } \rho)$. One family of spaces, $L^{2}\left(\varepsilon_{\rho}\right)$, is defined by the norm

$$
\|f\|=\left[\iint_{\varepsilon_{\rho}}|f(z)|^{2} d x d y\right]^{1 / 2}
$$

and the other, $H^{2}\left(\varepsilon_{\rho}\right)$, by the norm

$$
\|f\|=\left[\int_{\partial \varepsilon_{\rho}}|f(z)|\left|1-z^{2}\right|^{-1 / 2}|d z|\right]^{1 / 2} .
$$

Our main interest will be the asymptotic behavior of minimal CIR's in the two cases:

(a) $\rho \rightarrow \infty$,

(b) $\rho \rightarrow 1$.

In case (a), the two sets of minimal CIR's corresponding to $L^{2}\left(\varepsilon_{\rho}\right)$ and $H^{2}\left(\varepsilon_{\rho}\right)$ tend to the same limit, namely, to a rule which integrates as many monomials as possible, and if this number is less than $n+1$, to a rule which integrates the next monomial with a minimal error. In the first instance, we recover the classical Chebyshev rules. In the second, we arrive at GCIR's.

In case (b), we consider the asymptotic behavior of two classes of rules in each family of spaces:

(1) Minimal CIR's.

(2) CIR's minimal in the class of rules which integrate constants exactly, i.e., rules such that $w=2 / n$. This distinction in case (a) is of no interest since the limiting rule there belongs to class (2). The asymptotic behavior for $\rho \rightarrow 1$ is derived using some of the results in [7] which we quote without proof.

In Section 2, we formulate the problem and prove the existence of the GCIR's. In Section 3, we characterize the asymptotic minimal GCIR's for $\rho \rightarrow \infty$. In Section 4, we give an algebraic approach for computing the rules discussed in Section 3, for $n=8,10,11,12,13$. In Section 5 , we prove four theorems about the asymptotic behavior as $\rho \rightarrow 1$ of minimal GCIR's in $L^{2}\left(\varepsilon_{\rho}\right)$ and $H^{2}\left(\varepsilon_{\rho}\right)$. Finally, in Section 6, we give tables of the rules discussed in Section 4 and tables of minimal CIR's for $\rho$ approaching $1^{+}$to illustrate the theoretical results of Section 5 .

2. Formulation of the Problem. Let $\varepsilon_{\rho}$ designate the ellipse with foci at $( \pm 1,0)$, semimajor axis $a$ and semiminor axis $b=\left(a^{2}-1\right)^{1 / 2}$ where $\rho=(a+b)^{2}$. The two families of Hilbert spaces to be considered are the following:

(1) $L^{2}\left(\varepsilon_{\rho}\right)$-the collection of all functions $f(z)$ analytic in $\varepsilon_{\rho}, \rho>1$, such that

$$
\iint_{\mathcal{E}_{\rho}}|f(z)|^{2} d x d y<\infty,
$$

with the scalar product

$$
(f, g)=\iint_{\varepsilon_{\rho}} f(z) \overline{g(z)} d x d y
$$


(2) $H^{2}\left(\varepsilon_{\rho}\right)$-the collection of all functions $f(z)$ analytic in $\varepsilon_{\rho}, \rho>1$, such that

$$
\int_{\partial \varepsilon_{\rho}}|f(z)|^{2}\left|1-z^{2}\right|^{-1 / 2}|d z|<\infty,
$$

with the scalar product

$$
(f, g)=\int_{\partial \varepsilon_{\rho}} f(z) \overline{g(z)}\left|1-z^{2}\right|^{-1 / 2}|d z| .
$$

A complete orthonormal set $\left\{P_{m}(z)\right\}$ for $L^{2}\left(\varepsilon_{\rho}\right)$ is given by suitably normalized Chebyshev polynomials of the second kind [3, p. 241]:

$$
P_{m}(z)=\frac{2}{\sqrt{ } \pi}\left[\frac{m+1}{\rho^{m+1}-\rho^{-m-1}}\right]^{1 / 2} U_{m}(z), \quad m=0,1,2, \cdots,
$$

where $U_{m}(z)=\sin (m+1) \theta / \sin \theta$ with $z=\cos \theta$. For $H^{2}\left(\mathcal{E}_{\rho}\right)$, a complete orthonormal set $\left\{P_{m}(z)\right\}$ is given by suitably normalized Chebyshev polynomials of the first kind [3, p. 240]:

$$
\begin{aligned}
P_{m}(z) & =\sqrt{\frac{2}{\pi}}\left[\frac{1}{\rho^{m}+\rho^{-m}}\right]^{1 / 2} T_{m}(z), \quad m=1,2, \cdots, \\
P_{0}(z) & =1 /(2 \pi)^{1 / 2},
\end{aligned}
$$

where $T_{m}(z)=\cos n \theta$ with $z=\cos \theta$.

By the Riesz representation theorem, any bounded linear functional $L$ in a Hilbert space $H$ determines a unique element $l \in H$, called the representer of $L$, such that, for any $f \in H$,

$$
L(f)=(f, l) \text { and }\|L\|=\|l\| .
$$

The error in a given integration rule with abscissae in $[-1,1]$ is a bounded linear functional $E$ in both $L^{2}\left(\varepsilon_{\rho}\right)$ and $H^{2}\left(\varepsilon_{\rho}\right)$. Hence, there is a representer of this functional in each space. For a GCIR with abscissae $x_{1}, \cdots, x_{n}$ and weight $w$, the representer is

$$
r(z)=\phi(z)-\bar{w} \sum_{i=1}^{n} \phi_{x i}(z),
$$

where $\phi(z)$ is the representer of the linear functional

$$
\text { If }=\int_{-1}^{1} f(x) d x
$$

and $\phi_{x_{i}}(z)$ is the representer of the point functional

$$
L_{x_{i}} f=f\left(x_{i}\right) .
$$

Since in both spaces, convergence in norm implies pointwise convergence, the above representers can be expressed in terms of the complete orthonormal set:

$$
\begin{aligned}
\phi(z) & =\sum_{m=0}^{\infty}\left[\int_{-1}^{1} P_{m}(x) d x\right] P_{m}(z), \\
\phi_{x i}(z) & =\sum_{m=0}^{\infty} P_{m}\left(x_{i}\right) P_{m}(z)
\end{aligned}
$$




$$
r(z)=\sum_{m=0}^{\infty} \overline{\left[E\left(P_{m}\right)\right]} P_{m}(z) .
$$

For a given set of abscissae, the problem of determining the weight $w$ for which the error functional $E$ is of minimum norm is thus equivalent to the problem of best approximating the function $\phi(z)$ by the single function

$$
\psi(z)=\sum_{i=1}^{n} \phi_{x_{i}}(z)
$$

Hence, $w$ is determined by the condition:

$$
\left(\phi-\bar{w} \sum_{i=1}^{n} \phi_{x_{i}}, \sum_{i=1}^{n} \phi_{x_{i}}\right)=0
$$

and is given explicitly by:

$$
\bar{w}=\frac{\left(\phi, \sum_{i=1}^{n} \phi_{x_{i}}\right)}{\left(\sum_{i=1}^{n} \phi_{x_{i}}, \sum_{i=1}^{n} \phi_{x_{i}}\right)} .
$$

The norm of the corresponding error functional is given by

$$
\|E\|^{2}=\|r\|^{2}=(r, r)=(\phi, \phi)-w\left(\phi, \sum_{i=1}^{n} \phi_{x_{i}}\right) .
$$

By (1), (2), (3), (4), $w$ is a real continuous function of the $n$ abscissae as is, therefore, the norm of the corresponding error functional. Since a real continuous function attains its minimum value relative to a compact domain at a point therein, the existence of a point $\left(x_{1}, \cdots, x_{n}\right)$ in the hypercube $S_{n}:-1 \leqq x_{i} \leqq 1$ for which the norm of the corresponding error functional is a minimum is guaranteed.

Similar arguments prove the existence of a minimal GCIR in the class of rules which integrate constants exactly, since in this case $w=2 / n$, and the norm of the error functional is a real continuous function in $S_{n}$. We formulate these observations in the following theorem:

THEOREM 1. For any $\rho>1$, there exists an unconstrained minimal GCIR and a constrained minimal GCIR with $w=2 / n$ in each of the spaces $L^{2}\left(\varepsilon_{0}\right), H^{2}\left(\varepsilon_{\rho}\right)$.

We remark that although we have only been able to prove the existence of minimal GCIR's, nevertheless, in practice, these minimal rules turn out to be CIR's. We therefore conjecture that for every $\rho>1$, the minimal GCIR is indeed a CIR, both in the constrained and unconstrained cases in $L^{2}\left(\varepsilon_{\rho}\right)$ and $H^{2}\left(\varepsilon_{\rho}\right)$.

3. Asymptotic Behavior as $\rho \rightarrow \infty$. The following theorem characterizes the asymptotic behavior of GCIR's as $\rho \rightarrow \infty$ :

THEOREM 2. As $\rho \rightarrow \infty$, the minimal GCIR's with $n$ abscissae in both $L^{2}\left(\mathcal{E}_{\rho}\right)$ and $H^{2}\left(\mathcal{E}_{\rho}\right)$ tend to the same $G C I R, R_{n}$, with the following properties:

(1) $E_{n}\left(x^{i}\right)=0, i=0,1, \cdots, k-1$, for as large a value of $k$ as possible.

(2) If $k<n$, then $\left|E_{n}\left(x^{k}\right)\right|$ is minimal.

In particular, $w$ is given by $2 / n$.

Proof. The norm of the error functional is given, using (3), by:

$$
\|r\|^{2}=(r, r)=\sum_{m=0}^{\infty}\left|E_{m}\left(P_{m}\right)\right|^{2}=\sum_{m=0}^{\infty}\left[\int_{-1}^{1} P_{m}(x) d x-w \sum_{i=1}^{n} P_{m}\left(x_{i}\right)\right]^{2} .
$$


In $L^{2}\left(\varepsilon_{p}\right)$, this norm has the form:

$$
\left\|r_{L}\right\|^{2}=\frac{4}{\pi} \sum_{m=0}^{\infty}\left[\frac{m+1}{\rho^{m+1}-\rho^{-m-1}} E_{n}\left(U_{m}\right)\right]^{2}
$$

and in $H^{2}\left(\varepsilon_{p}\right)$, the form

$$
\left\|r_{H}\right\|^{2}=\frac{2}{\pi} \sum_{m=0}^{\infty},\left[\frac{1}{\rho^{m}+\rho^{-m}} E_{n}\left(T_{m}\right)\right]^{2},
$$

where the prime indicates that the term for $m=0$ is to be halved.

For $\rho \rightarrow \infty$, the asymptotic expressions of the two norms are:

$$
\begin{aligned}
& \left\|r_{L}\right\|=\left(\frac{4(k+1)}{\pi\left(1-\rho^{-2 k-2}\right)}\right)^{1 / 2}\left|E_{n}\left(U_{k}\right)\right| \rho^{-(k+1) / 2}+O\left(\rho^{-(k+2) / 2}\right), \\
& \left\|r_{H}\right\|=\left(\frac{2}{\pi\left(1+\rho^{-2 k}\right)}\right)^{1 / 2}\left|E_{n}\left(T_{k}\right)\right| \rho^{-k / 2}+O\left(\rho^{-(k+1) / 2}\right),
\end{aligned}
$$

where $k$ is the degree of the first polynomial in the orthonormal sequence which is not integrated exactly. Now the larger $k$ is, the smaller $\|r\|$ becomes. Hence the asymptotic rule $R_{n}$, which minimizes $\|r\|$, integrates as many polynomials in the sequence as possible. If $k<n+1$, there are $n-k+1$ free parameters at our disposal which are used so as to minimize $\left|E_{n}\left(P_{k}\right)\right|$.

Now, the conditions $E_{n}\left(P_{i}\right)=0, j=0,1, \cdots, k-1$, are equivalent to the conditions $E_{n}\left(x^{i}\right)=0, j=0,1, \cdots, k-1$. This implies that the parameter set which minimizes $\left|E_{n}\left(P_{k}\right)\right|$ subject to the constraints $E_{n}\left(P_{i}\right)=0, j=0,1, \cdots, k-1$, is identical to the set which minimizes $\left|E_{n}\left(x^{k}\right)\right|$ subject to the constraints $E_{n}\left(x^{i}\right)=$ $0, j=0,1, \cdots, k-1$. Hence, the resulting rule is of degree $k-1$. By choosing $w=2 / n$, we are assured that $k>0$.

Theorem 2 gives an algebraic characterization of the asymptotic rule, which suggests an algebraic approach to the problem of determining such rules explicitly for various values of $n$. Another possibility is to compute minimal GCIR's for a sequence of monotonically increasing values of $\rho$, by minimizing the norm of the error functional as a function of the weight and abscissae. This numerical approach failed for those values of $n$ for which classical rules do not exist, since the norms have many local minima, corresponding to rules which are exact for the maximal number of monomials.

4. The Algebraic Approach. The abscissae, $x_{i}, i=1, \cdots, n$, and weight $w$ of the asymptotic GCIR, for $\rho \rightarrow \infty$, satisfy the following conditions:

$$
w \sum_{i=1}^{n} x_{i}^{j}=\int_{-1}^{1} x^{i} d x=\frac{1+(-1)^{i}}{j+1}, \quad j=0,1, \cdots, k-1,
$$

and the corresponding rule $R_{n}$ is of degree $k-1$. Taking $w=2 / n$ to satisfy the condition for $j=0$, we rewrite the other conditions in the form

$$
\sum_{i=1}^{n} x_{i}^{i}=\frac{n}{2(j+1)}\left[1+(-1)^{i}\right] \equiv \alpha_{i}, \quad j=1, \cdots, k-1 .
$$


Let

$$
Q_{n}(x)=\prod_{i=1}^{n}\left(x-x_{i}\right) \equiv \sum_{i=0}^{n} a_{n-i} x^{i}, \quad a_{0}=1,
$$

then $a_{0}, a_{1}, \cdots, a_{k-1}$ satisfy the following recurrence formula [6, p. 356]

$$
r a_{r}+\alpha_{1} a_{r-1}+\alpha_{2} a_{r-2}+\cdots+\alpha_{r-1} a_{0}+\alpha_{r}=0, \quad r=1, \cdots, k-1 .
$$

For the case $k=n+1, a_{1}, \cdots, a_{n}$ are determined by (7) and the roots of $Q_{n}(x)$ are the abscissae of the integration rule. Since $\sum_{i=0}^{n} x_{i}^{i}=0$ for $j$ odd, the resulting rule is symmetric.

For nonclassical rules which occur when the roots of $Q_{n}(x)$ are not all real, we have that $k<n+1$ and the following considerations apply:

Let $\tilde{a}_{1}, \cdots, \tilde{a}_{n}$ denote the coefficients obtained using (6) and (7). In order that $k$ be as large as possible, we must retain as many coefficients as possible in the above sequence, at the same time ensuring that the polynomial $Q_{n}(x)$ possesses $n$ real roots, preferably in $[-1,1]$.

For a rule of degree $k-1$, the corresponding polynomial $Q_{n}(x)$ is a function of the $n+1-k$ parameters $a_{k}, \cdots, a_{n}$, namely:

$$
Q_{n}(x)=Q_{n}\left(x ; a_{k}, \cdots, a_{n}\right)=x^{n}+\sum_{i=1}^{k-1} \tilde{a}_{i} x^{n-i}+\sum_{i=k}^{n} a_{i} x^{n-i} .
$$

The parameters $a_{k}, \cdots, a_{n}$ are to be chosen to satisfy the following two requirements:

(1) $Q_{n}(x)$ has $n$ real roots,

(2) $\left|E_{n}\left(x^{k}\right)\right|$ is a minimum.

Using (7) to express $\sum_{i=1}^{n} x_{i}^{k}$ in terms of the coefficients of $Q_{n}(x)$, we write $\left|E_{n}\left(x^{k}\right)\right|$ as follows:

$$
\left|E_{n}\left(x^{k}\right)\right|=\left|\frac{1+(-1)^{k}}{k+1}-\frac{2}{n} \sum_{i=1}^{n} x_{i}^{k}\right|=\left|\frac{1+(-1)^{k}}{k+1}-\frac{2}{n} \sum_{i=1}^{k-1} \alpha_{i} \tilde{a}_{k-1}+2 a_{k}\right|,
$$

where $\alpha_{1}, \cdots, \alpha_{k-1}$ are defined by (6). Thus, $\left|E_{n}\left(x^{k}\right)\right|$ is independent of $a_{k+1}, \cdots, a_{n}$ and, since the right-hand side of the above equation vanishes for $a_{k}=\tilde{a}_{k}$, we see that the closer $a_{k}$ is to $\tilde{a}_{k}$, the smaller $\left|E_{n}\left(x^{k}\right)\right|$ will be.

Since $k$ is not known a priori, we proceed as follows: For $n$ even, we first set $k=n$, so that $Q_{n}(x)=Q_{n}\left(x, a_{n}\right)$ and $Q_{n}^{\prime}(x)$ is independent of $a_{n}$. Let $\zeta_{1}, \cdots, \zeta_{m}$ be the (not necessarily distinct) real roots of $Q_{n}^{\prime}(x)$. The following two conditions are necessary and sufficient that there exist $a_{n}$ such that $Q_{n}(x)=x^{n}+\sum_{i=1}^{n-1} \tilde{a}_{i} x^{n-i}+$ $a_{n}$ possess $n$ real roots:

$$
\begin{aligned}
& \text { (a) } m=n-1 \\
& \text { (b) } r \equiv \max _{1 \leq i \leq m ; Q_{n}^{\prime \prime \prime}\left(\zeta_{i}\right)>0} Q_{n}\left(\zeta_{i}, 0\right) \leqq \min _{1 \leq i \leq m ; Q_{*}^{\prime \prime \prime}\left(\zeta_{i}\right)<0} Q_{n}\left(\zeta_{i}, 0\right) \equiv s \text {. }
\end{aligned}
$$

(This can be seen most clearly from the graph of $Q_{n}(x)$.) Condition (a) holds for $n=8,10,12$ while condition (b) holds only for $n=8,10$.

When both (a) and (b) hold, the $a_{n}$ which lies in $[-s,-r]$ and is closest to $\tilde{a}_{n}$ is the required value which we denote by $a_{n}^{*}$. Since $\tilde{\alpha}_{n} \notin[-s,-r]$, it follows that $a_{n}^{*}$ is one of the endpoints of $[-s,-r]$ and yields a double root for $Q_{n}(x)$. Since $Q_{n}(x)$ is a symmetric polynomial, the rule $R_{n}$ will have one double node when the double 
root of $Q_{n}\left(x ; a_{n}^{*}\right)$ is at $x=0(n=8)$ and two double nodes, otherwise $(n=10)$.

For $n=12, k<n$ since $s<r$. Now $k=n-1$ is not possible since any change in $a_{n-1}$ is equivalent to the addition of a linear function to the even function $Q_{n}\left(x ; \tilde{a}_{n-1}, 0\right)$ and this operation cannot reverse the relation $s<r$. Hence, the next trial value is $k=n-2$, which corresponds to a change in $a_{n-2}$. Since for $\tilde{a}_{n-2}, s-r<0$, we take $a_{n-2}^{*}$ to be the closest value to $\tilde{a}_{n-2}$ for which $s-r \geqq 0$, where $r, s$ correspond to $Q_{n}\left(x ; a_{n-2}, 0,0\right)$. For this value of $a_{n-2}^{*}, r=s, a_{n}^{*}$ is taken to be $-s$ and the resulting $R_{n}$ has two double nodes in each half-interval, since it is symmetric.

The procedure for $n$ odd is similar, but starts with $k=n-1$, since if $k=n+1$ is rejected, then $k=n$ cannot hold because, in this case, $s=-r<0$. For $n=11$, 13, $Q_{n}^{\prime \prime}(x)$ has $n-2$ roots in $[-1,1]$ and $r_{1}<s_{1}$ where $r_{1}$ and $s_{1}$ are the analogues of $r$ and $s$ with respect to $Q_{n}^{\prime}(x ; 0)$. By confining $a_{n-1}^{*}$ to the interval $\left[-s_{1},-r_{1}\right]$, we guarantee that $Q_{n}^{\prime}\left(x ; a_{n-1}^{*}\right)$ has $n-1$ roots in $[-1,1]$ and thus it remains to choose $a_{n-1}^{*}$ to be the closest value to $\tilde{a}_{n-1}$ in $\left[-s_{1},-r_{1}\right]$, subject to the condition that $s-$ $r \geqq 0$. Since this occurs when $s-r=0$, we found $a_{n-1}^{*}$ by treating $s-r$ as a function of $a_{n-1}$ and using the method of bisection to obtain the desired root.

The resulting polynomial $Q_{n}\left(x ; a_{n-1}^{*}, 0\right)$ is odd so that $r=-s$ and since $s-$ $r=0$, we have that $r=s=0$ and hence $a_{n}^{*}=0$. Thus, the integration rule is symmetric with one double node in each half-interval.

In Table 2, we give the abscissae of the five rules computed for $n=8,10,11,12,13$. Since all the rules are symmetric, only nonnegative abscissae are tabulated.

5. Asymptotic Behavior as $\rho \rightarrow 1$.

(I) The Spaces $L^{2}\left(\varepsilon_{\rho}\right), \rho>1$. In this fanily of spaces, the representers (3) can be written explicitly, using (1) as:

$$
\begin{aligned}
\phi(z) & =\frac{8}{\pi} \sum_{m=0}^{\infty}\left(\rho^{2 m+1}-\rho^{-2 m-1}\right)^{-1} U_{2 m}(z), \\
\phi_{x_{i}}(z) & =\frac{4}{\pi} \sum_{m=0}^{\infty}(m+1)\left(\rho^{m+1}-\rho^{-m-1}\right) U_{m}\left(x_{i}\right) U_{m}(z) .
\end{aligned}
$$

Using the following result derived in [7]:

For $\rho \rightarrow 1^{+}$and $-1<x_{i}, x_{i}<1$,

$$
\begin{aligned}
(\rho-1 / \rho) \sum_{m=0}^{\infty} 2\left(\rho^{2 m+1}-\rho^{-2 m-1}\right)^{-1} U_{2 m}\left(x_{i}\right) & =\frac{\pi}{2}\left(1-x_{i}^{2}\right)^{-1 / 2}+o(1), \\
(\rho-1 / \rho) \sum_{m=0}^{\infty}(m+1)\left(\rho^{m+1}-\rho^{-m-1}\right)^{-1} U_{m}\left(x_{i}\right) U_{m}\left(x_{i}\right) & =\frac{\pi^{2}}{4} \delta_{i j}\left(1-x_{i}^{2}\right)^{-1}+o(1),
\end{aligned}
$$

we find the asymptotic behavior of the following scalar products, for $-1<x_{i}, x_{i}<1$ :

$$
\begin{gathered}
\left(\rho-\frac{1}{\rho}\right)\left(\phi, \phi_{x_{i}}\right)=\left(\rho-\frac{1}{\rho}\right) \phi\left(x_{i}\right)=2\left(1-x_{i}^{2}\right)^{-1 / 2}+o(1), \\
\left(\rho-\frac{1}{\rho}\right)^{2}\left(\phi_{x_{i}}, \phi_{x_{i}}\right)=\left(\rho-\frac{1}{\rho}\right)^{2} \phi_{x_{i}}\left(x_{i}\right)=\pi \delta_{i j}\left(1-x_{i}^{2}\right)^{-1}+o(1) .
\end{gathered}
$$

Inserting these expressions into (4), we find the minimizing weight $w$ corresponding 
to a given set of abscissae $x_{1}, \cdots, x_{n}$ in $(-1,1)$ to be:

$$
w=\frac{\left(\phi, \sum_{i=1}^{n} \phi_{x_{i}}\right)}{\left(\sum_{i=1}^{n} \phi_{x_{i}}, \sum_{i=1}^{n} \phi_{x i}\right)}=\left[\frac{2}{\pi} \frac{\sum_{i=1}^{n}\left(1-x_{i}^{2}\right)^{-1 / 2}}{\sum_{i=1}^{n}\left(1-x_{i}^{2}\right)^{-1}}+o(1)\right]\left(\rho-\frac{1}{\rho}\right) .
$$

By (5) and (11), the norm of the corresponding error functional is given by

$$
(r, r)=(\phi, \phi)-\frac{4}{\pi} \frac{\left[\sum_{i=1}^{n}\left(1-x_{i}^{2}\right)^{-1 / 2}\right]^{2}}{\sum_{i=1}^{n}\left(1-x_{i}^{2}\right)^{-1}}+o(1)
$$

with

$$
(\phi, \phi)=\int_{-1}^{1} \phi(x) d x=\left(\rho-\frac{1}{\rho}\right)^{-1}[2 \pi+o(1)] .
$$

The set of abscissae of the asymptotic minimal rule is clearly that set for which

$$
\frac{\left[\sum_{i=1}^{n}\left(1-x_{i}^{2}\right)^{-1 / 2}\right]^{2}}{\sum_{i=1}^{n}\left(1-x_{i}^{2}\right)^{-1}}
$$

is maximal. By the Cauchy-Schwarz inequality, we have that

$$
\frac{\left[\sum_{i=1}^{n}\left(1-x_{i}^{2}\right)^{-1 / 2}\right]^{2}}{\sum_{i=1}^{n}\left(1-x_{i}^{2}\right)^{-1}} \leqq \frac{n \sum_{i=1}^{n}\left(1-x_{i}^{2}\right)^{-1}}{\sum_{i=1}^{n}\left(1-x_{i}^{2}\right)^{-1}}=n
$$

and equality is possible only for $x_{i}= \pm \eta, i=1, \cdots, n$, for a fixed value $\eta$ with $|\eta|<1$. These results yield the following theorem:

THEOREM 3. For a set of $n$ abscissae in $(-1,1)$, the minimizing weight $w$ of the corresponding GCIR tends to zero, as $\rho \rightarrow 1$, according to (12) and the norm of the error functional of this GCIR is asymptotically $\|\phi\|$, where

$$
\|\phi\|=\left(\rho-\frac{1}{\rho}\right)^{-1 / 2}\left[(2 \pi)^{1 / 2}+o(1)\right] .
$$

All the abscissae of the minimal GCIR tend, as $\rho \rightarrow 1$, to a single point or two symmetric points, but the location of these points is unknown. The amount by which $(r, r)$ is reduced per abscissa is $4 / \pi+o(1)$ which is negligible in the limit.

Remark. This latter quantity is identical with the asymptotic amount by which any abscissa in the general minimal integration rule reduces $(r, r)$ [7].

For a GCIR with $w=2 / n$, the norm of the error functional is given by:

$$
\begin{aligned}
(r, r)= & (\phi, \phi)-\frac{4}{n} \sum_{i=1}^{n}\left(\phi, \phi_{x_{i}}\right)+\frac{4}{n^{2}} \sum_{i, j=1}^{n}\left(\phi_{x_{i}}, \phi_{x_{i}}\right) \\
= & {\left[2 \pi-\frac{8}{n} \sum_{i=1}^{n}\left(1-x_{i}^{2}\right)^{-1 / 2}+o(1)\right]\left(\rho-\frac{1}{\rho}\right)^{-1} } \\
& +\left[\frac{4 \pi}{n^{2}} \sum_{i=1}^{n}\left(1-x_{i}^{2}\right)^{-1}+o(1)\right]\left(\rho-\frac{1}{\rho}\right)^{-2} .
\end{aligned}
$$

This yields the corresponding theorem.

THEOREM 4. The abscissae in the minimal GCIR, constrained by $w=2 / n$, tend to zero as $\rho \rightarrow 1$. Each point in this asymptotic rule contributes 


$$
\left[\frac{4 \pi}{n^{2}}+o(1)\right]\left(\rho-\frac{1}{\rho}\right)^{-2}
$$

to the value of $(r, r)$.

(II) The Spaces $H^{2}\left(\mathcal{E}_{p}\right), \rho>1$. In this family of spaces, the representers (3) can be written explicitly using (2) as:

$$
\begin{aligned}
\phi(z) & =\frac{4}{\pi} \sum_{m=0}^{\infty}\left[\left(1-4 m^{2}\right)\left(\rho^{2 m}+\rho^{-2 m}\right)\right]^{-1} T_{2 m}(z), \\
\phi_{x_{i}}(z) & =\frac{2}{\pi} \sum_{m=0}^{\infty}\left(\rho^{m}+\rho^{-m}\right)^{-1} T_{m}\left(x_{i}\right) T_{m}(z) .
\end{aligned}
$$

Using the following result derived in [7]:

For $\rho \rightarrow 1^{+}$and $-1<x_{i}, x_{i}<1$,

$$
\begin{aligned}
& \sum_{m=0}^{\infty}\left(1-4 m^{2}\right)^{-1}\left(\rho^{2 m}+\rho^{-2 m}\right)^{-1} T_{2 m}\left(x_{i}\right)=\frac{\pi}{8}\left(1-x_{i}^{2}\right)^{1 / 2}+o(1), \\
& \left(\rho-\frac{1}{\rho}\right) \sum_{m=0}^{\infty}\left(\rho^{m}+\rho^{-m}\right)^{-1} T_{m}\left(x_{i}\right) T_{m}\left(x_{i}\right)=\frac{\pi}{4} \delta_{i j}+o(1),
\end{aligned}
$$

we find the asymptotic behavior of the following scalar products for $-1<x_{i}, x_{i}<1$ :

$$
\begin{aligned}
\left(\phi, \phi_{x_{i}}\right) & =\phi\left(x_{i}\right)=\frac{1}{2}\left(1-x_{i}^{2}\right)^{1 / 2}+o(1), \\
\left(\rho-\frac{1}{\rho}\right)\left(\phi_{x_{i}}, \phi_{x_{i}}\right) & =\left(\rho-\frac{1}{\rho}\right) \phi_{x_{i}}\left(x_{i}\right)=\frac{1}{2} \delta_{i j}+o(1) .
\end{aligned}
$$

Inserting these expressions into (4), we find the minimizing weight $w$ corresponding to a given set of abscissae $x_{1}, \cdots, x_{n}$ in $(-1,1)$ to be:

$$
w=\frac{\left(\phi, \sum_{i-1}^{n} \phi_{x_{i}}\right)}{\left(\sum_{i=1}^{n} \phi_{x_{i}}, \sum_{i=1}^{n} \phi_{x_{i}}\right)}=\left[\frac{1}{n} \sum_{i=1}^{n}\left(1-x_{i}^{2}\right)^{1 / 2}+o(1)\right]\left(\rho-\frac{1}{\rho}\right) .
$$

By (5) and (15), the norm of the corresponding error functional is given by

$$
(r, r)=(\phi, \phi)-\left[\frac{1}{2 n}\left(\sum_{i=1}^{n}\left(1-x_{i}^{2}\right)^{1 / 2}\right)^{2}+o(1)\right]\left(\rho-\frac{1}{\rho}\right),
$$

with $(\phi, \phi)=\int_{-1}^{1} \phi(x) d x=\pi / 4+o(1)$.

By (17), the abscissae of the minimal GCIR's tend to zero since the choice $x_{i}=$ $0, i=1, \cdots, n$, maximizes the quantity

$$
\frac{1}{2 n}\left(\sum_{i=1}^{n}\left(1-x_{i}^{2}\right)^{1 / 2}\right)^{2} .
$$

These results yield the following theorem:

THEOREM 5. For a set of $n$ abscissae in $(-1,1)$, the minimizing weight $w$ of the corresponding GCIR tends to zero, as $\rho \rightarrow 1$, according to (16) and the norm of the error functional of this GCIR tends to $\pi / 4$. All the abscissae of the minimal GCIR tend to zero as $\rho \rightarrow 1$ and the amount by which $(r, r)$ is reduced per abscissa is asymptotically $\frac{1}{2}(\rho-1 / \rho)$ which is negligible in the limit.

Remark. This latter quantity is identical with the asymptotic amount by which any abscissa in the general minimal integration rule reduces $(r, r)$ [7]. 


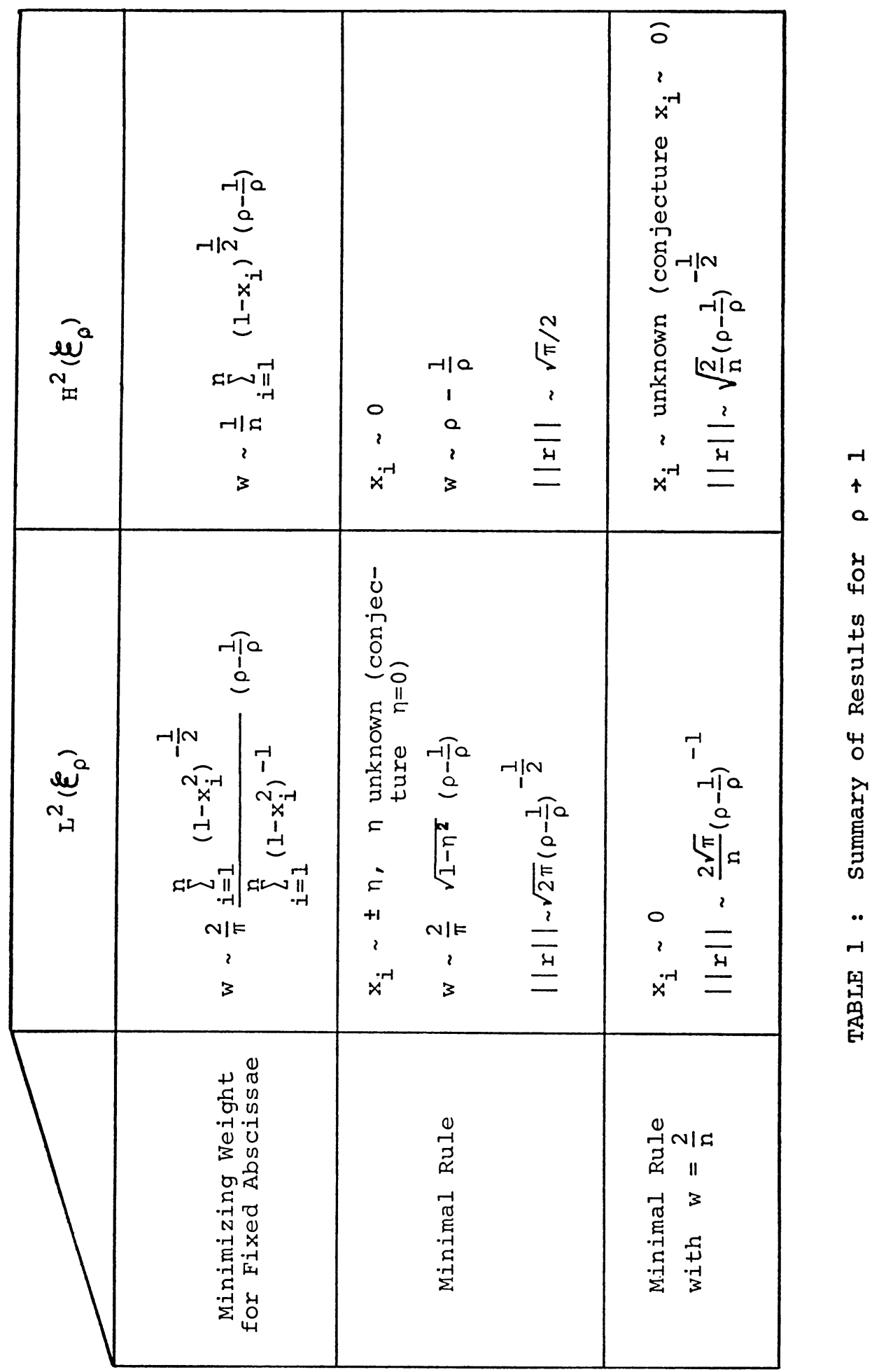




\begin{tabular}{|c|c|c|c|c|c|c|c|}
\hline$\overline{\widetilde{x}}$ & 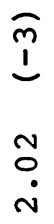 & $\begin{array}{l}\hat{m} \\
1 \\
\stackrel{-}{r} \\
\stackrel{-1}{-1}\end{array}$ & $\begin{array}{l}\stackrel{7}{1} \\
\stackrel{1}{N} \\
\stackrel{1}{*}\end{array}$ & & $\begin{array}{l}6 \\
1 \\
2 \\
\stackrel{9}{+} \\
\dot{1}\end{array}$ & $\begin{array}{l}\stackrel{+}{L} \\
\dot{L} \\
\dot{\infty} \\
\dot{m}\end{array}$ & \\
\hline $\begin{array}{l}\text { o } \\
0 \\
0 \\
0 \\
z\end{array}$ & 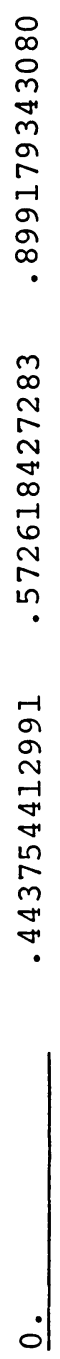 & 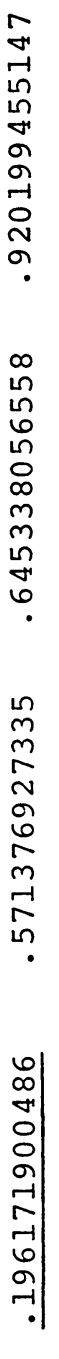 & 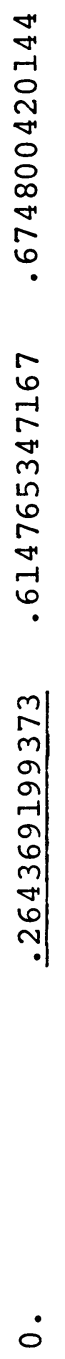 & 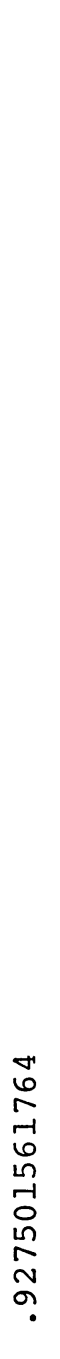 & 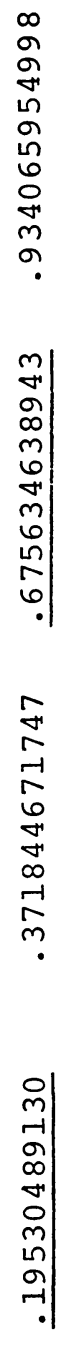 & 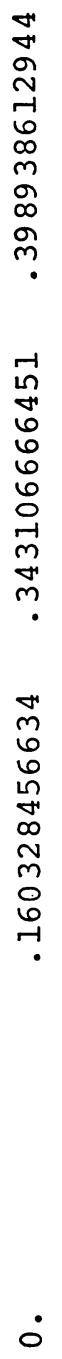 & 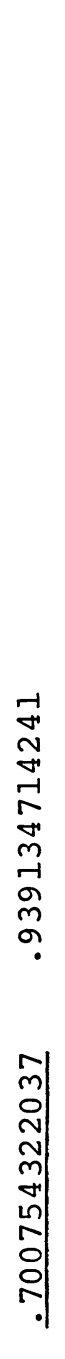 \\
\hline$y$ & $\infty$ & 엄 & 억 & & $\underset{-1}{\circ}$ & $\stackrel{N}{r}$ & \\
\hline G & $\infty$ & 욱 & $\underset{-1}{-1}$ & & $\underset{r}{N}$ & $\underset{r}{m}$ & \\
\hline
\end{tabular}




\begin{tabular}{|c|c|c|c|c|c|c|c|c|}
\hline \multirow{4}{*}{6} & \multirow{3}{*}{$\begin{array}{l}0 \\
0 \\
0 \\
0 \\
-1 \\
U \\
U \\
\mathcal{Q}\end{array}$} & 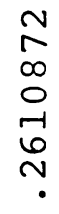 & $\begin{array}{l}m \\
\sim \\
\sim \\
\text { on } \\
\sim \\
\sim \\
\sim\end{array}$ & $\begin{array}{l}0 \\
\text { r } \\
\text { m } \\
0 \\
0 \\
\text { م } \\
\text { r-1 }\end{array}$ & $\begin{array}{l}\text { r } \\
\text { r } \\
\text { a } \\
\text { a } \\
\text { r } \\
\text { r }\end{array}$ & 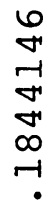 & $\begin{array}{l}\stackrel{0}{m} \\
6 \\
\overrightarrow{-1} \\
m \\
\stackrel{-1}{-1} \\
\cdot\end{array}$ & 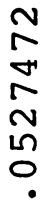 \\
\hline & & 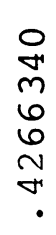 & 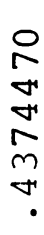 & 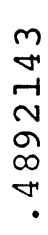 & $\begin{array}{l}\text { 두 } \\
\text { a } \\
6 \\
m \\
m \\
\text { in }\end{array}$ & $\begin{array}{l}0 \\
\text { ฟ' } \\
\text { o } \\
\text { N } \\
\text { m } \\
\text { in } \\
\text { ? }\end{array}$ & $\begin{array}{l}\text { r } \\
\text { n } \\
\text { r } \\
0 \\
\text { m } \\
m \\
\text {. }\end{array}$ & 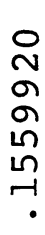 \\
\hline & & 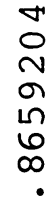 & \begin{tabular}{l}
6 \\
Ln \\
$\sigma$ \\
$\sigma$ \\
\multirow{1}{*}{} \\
6 \\
$\infty$
\end{tabular} & $\begin{array}{l}N \\
\mho \\
0 \\
+ \\
m \\
\dot{H} \\
\infty \\
.\end{array}$ & $\begin{array}{l}6 \\
0 \\
m \\
0 \\
1 \\
6 \\
\infty \\
.\end{array}$ & $\begin{array}{l}\tilde{H} \\
\\
\stackrel{0}{N} \\
\underset{N}{\infty} \\
\infty\end{array}$ & 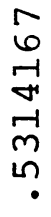 & $\begin{array}{l}N \\
\sigma \\
r \\
6 \\
6 \\
\text { Ln } \\
\sim\end{array}$ \\
\hline & 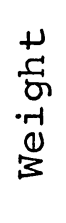 & $\begin{array}{l}m \\
m \\
m \\
m \\
m \\
m \\
m\end{array}$ & $\begin{array}{l}q \\
\text { m } \\
m \\
m \\
m \\
m \\
m \\
m\end{array}$ & 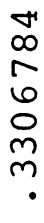 & $\begin{array}{l}-1 \\
m \\
0 \\
m \\
N \\
0 \\
m \\
m\end{array}$ & 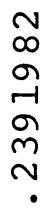 & 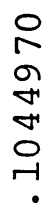 & $\begin{array}{l}\text { r } \\
\text { m } \\
\text { r } \\
\text { m } \\
0 \\
0\end{array}$ \\
\hline \multirow{2}{*}{$m$} & $\begin{array}{l}0 \\
\text { Un } \\
\text { Un } \\
\cdot-1 \\
U \\
\text { Un } \\
\text { Uे }\end{array}$ & 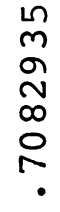 & $\begin{array}{l}r \\
\infty \\
m \\
6 \\
r \\
r \\
r \\
\end{array}$ & $\begin{array}{l}m \\
0 \\
0 \\
\\
\text { r-1 } \\
\stackrel{N}{N} \\
\text {. }\end{array}$ & \begin{tabular}{l}
$n$ \\
\multirow{n}{*}{} \\
$m$ \\
$\infty$ \\
$\sim$ \\
$\sigma$ \\
$\sigma$
\end{tabular} & 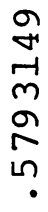 & & 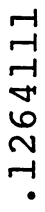 \\
\hline & $\begin{array}{l}+\underset{c}{+} \\
\text { G } \\
\cdot-1 \\
0 \\
3\end{array}$ & $\begin{array}{l}\text { 더 } \\
m \\
6 \\
6 \\
6 \\
0 \\
.\end{array}$ & 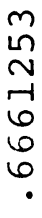 & 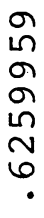 & 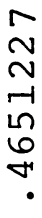 & $\begin{array}{l}0 \\
\infty \\
0 \\
m \\
\infty \\
0 \\
m \\
\text {. }\end{array}$ & & $\begin{array}{l}0 \\
\text { o } \\
\text {-1 } \\
\infty \\
\text { in } \\
\text { m } \\
0\end{array}$ \\
\hline 10 & & $\stackrel{0}{\dot{v}}$ & • & $\vec{H}$ & $\begin{array}{l}m \\
0 \\
\dot{H}\end{array}$ & $\begin{array}{r}-1 \\
0 \\
-1\end{array}$ & $\begin{array}{l}\text { 겅 } \\
\text { ᄋ } \\
0 \\
-1\end{array}$ & $\begin{array}{l}\text {-1 } \\
\text { ○ } \\
0 \\
0 \\
0 \\
\text { - }\end{array}$ \\
\hline
\end{tabular}

$\overbrace{n}^{a}$

.

$\stackrel{n}{0}$

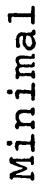

m 


\begin{tabular}{|c|c|c|c|c|c|c|c|c|}
\hline \multirow{4}{*}{6} & \multirow{3}{*}{$\begin{array}{l}0 \\
0 \\
02 \\
02 \\
-1 \\
0 \\
02 \\
0 \\
0\end{array}$} & 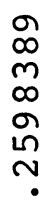 & 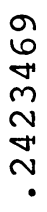 & 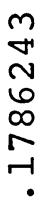 & $\begin{array}{l}0 \\
\infty \\
6 \\
1 \\
1 \\
0 \\
6 \\
-1\end{array}$ & 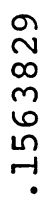 & 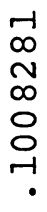 & \begin{tabular}{l} 
ro \\
$\infty$ \\
$\circ$ \\
$m$ \\
o \\
\multirow{\gamma}{*}{} \\
0
\end{tabular} \\
\hline & & 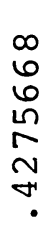 & 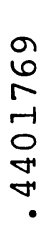 & $\begin{array}{l}\infty \\
\sigma \\
\sim \\
\sim \\
\stackrel{+}{ } \\
\infty \\
\sigma^{\prime} \\
\bullet\end{array}$ & 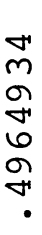 & $\begin{array}{l}\sigma \\
1 \\
+1 \\
\infty \\
\infty \\
0 \\
\forall \\
+\end{array}$ & 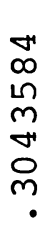 & 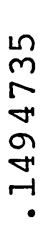 \\
\hline & & 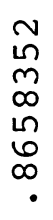 & 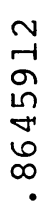 & 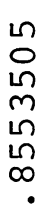 & $\begin{array}{l}\stackrel{N}{\infty} \\
\sim \\
\sim \\
N \\
\stackrel{N}{m} \\
\infty \\
.\end{array}$ & $\begin{array}{l}r \\
\text { on } \\
\infty \\
N \\
N \\
\\
\end{array}$ & $\begin{array}{l}m \\
m \\
\sigma \\
1 \\
6 \\
\text { '-1 } \\
\text { in }\end{array}$ & $\begin{array}{l}m \\
6 \\
\infty \\
0 \\
6 \\
\llcorner \\
\sim \\
\text { ம }\end{array}$ \\
\hline & 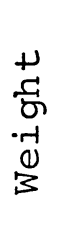 & $\begin{array}{l}m \\
m \\
m \\
m \\
m \\
m \\
m\end{array}$ & $\begin{array}{l}\text { N } \\
m \\
m \\
m \\
m \\
m\end{array}$ & $\begin{array}{l}m \\
\stackrel{m}{N} \\
\stackrel{0}{0} \\
m \\
m \\
m\end{array}$ & 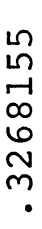 & 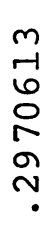 & 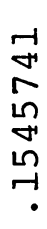 & $\begin{array}{l}\pi \\
1 \\
0 \\
\infty \\
0 \\
0 \\
10 \\
0 \\
0\end{array}$ \\
\hline \multirow{2}{*}{$m$} & $\begin{array}{l}\sigma \\
U \\
U \\
U-1 \\
U \\
U \\
0 \\
0\end{array}$ & 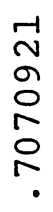 & \begin{tabular}{l}
$\infty$ \\
$N$ \\
\multirow{1}{*}{} \\
$\infty$ \\
0 \\
0 \\
0
\end{tabular} & \begin{tabular}{l}
$m$ \\
$\infty$ \\
0 \\
\multirow{+}{1}{} \\
$\infty$ \\
$\infty$ \\
6
\end{tabular} & $\begin{array}{l}m \\
\infty \\
+1 \\
\circ \\
\infty \\
0 \\
6\end{array}$ & 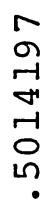 & 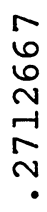 & $\begin{array}{l}\stackrel{\circ}{\sigma} \\
\text { N } \\
\underset{N}{N} \\
\underset{\sim}{N} \\
\text { r }\end{array}$ \\
\hline & 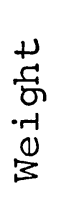 & $\begin{array}{l}\sim \\
0 \\
6 \\
6 \\
6 \\
0 \\
6\end{array}$ & 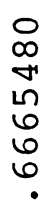 & 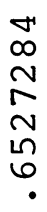 & 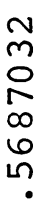 & 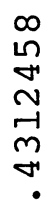 & 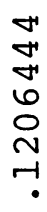 & $\begin{array}{l}0 \\
\text { م } \\
\text { o } \\
0 \\
6 \\
\text { 1n } \\
0 \\
\text {. }\end{array}$ \\
\hline$\sigma$ & & $\begin{array}{l}0 \\
\dot{N}\end{array}$ & ?ח & - & $\begin{array}{l}m \\
0 \\
-1\end{array}$ & $\begin{array}{l}-1 \\
0 \\
-1\end{array}$ & $\begin{array}{l}-1 \\
0 \\
0 \\
0\end{array}$ & $\begin{array}{l}-1 \\
0 \\
0 \\
0 \\
0 \\
-1\end{array}$ \\
\hline
\end{tabular}

Uூ 


\begin{tabular}{|l|c|c|c|c|}
\hline a & \multicolumn{3}{|c|}{ Abscissae } \\
\hline 2.0 & Abscissa & \multicolumn{3}{|c|}{6} \\
1.5 & .7082893 & .8659204 & .4266340 & .2610872 \\
1.1 & .7115646 & .8649953 & .4374460 & .2459727 \\
1.03 & .6165277 & .8558165 & .5212156 & .1745388 \\
1.01 & .4359713 & .7698833 & .4736271 & .1595859 \\
1.001 & .1906934 & .3986599 & .2348115 & .0777661 \\
1.0001 & .0781855 & .1712035 & .1003360 & .0331459 \\
\hline
\end{tabular}

TABLE 5 : Minimal CIR's with $w=\frac{2}{n}$ in $L^{2}\left(\varepsilon_{\rho}\right)$

\begin{tabular}{|l|c|c|c|c|}
\hline a n & 3 & \multicolumn{3}{|c|}{6} \\
\hline & Abscissa & \multicolumn{3}{|c|}{ Abscissae } \\
\hline 2.0 & .7070938 & .8658352 & .4275668 & .2598389 \\
1.5 & .7068802 & .8645912 & .4401770 & .2423468 \\
1.1 & .6916330 & .8555244 & .4856001 & .1786628 \\
1.03 & .6351125 & .8394149 & .5000705 & .1668131 \\
1.01 & .5519835 & .7984626 & .4858830 & .1626058 \\
1.001 & .3338414 & .5895055 & .3534554 & .1179710 \\
1.0001 & .1613753 & .3232849 & .1309153 & .0632936 \\
\hline
\end{tabular}

TABLE 6 : Minimal CIR's with $w=\frac{2}{n}$ in $H^{2}\left(\varepsilon_{\rho}\right)$ 
For a GCIR with $w=2 / n$, the norm of the error functional is given by:

$$
(r, r)=\frac{\pi}{4}-\frac{2}{n} \sum_{i=1}^{n}\left(1-x_{i}^{2}\right)^{1 / 2}+o(1)+\left[\frac{2}{n}+o(1)\right]\left(\rho-\frac{1}{\rho}\right)^{-1} .
$$

This yields the corresponding theorem:

THEOREM 6. For the minimization of $(r, r)$, all sets of $n$ abscissae in $(-1,1)$ are equally good insofar as the highest order term in $(\rho-1 / \rho)^{-1}$ is concerned. Any such set increases $(r, r)$ asymptotically by $(2 / n)(\rho-1 / \rho)^{-1}$.

We summarize the results of this section in Table 1 .

6. Tables. In Table 2, asymptotic GCIR's as $\rho \rightarrow \infty$ are given for $n=8,10,11$, 12,13 . The weight $w$ is $2 / n$. Since the rules are symmetric, only nonnegative abscissae are given. Double nodes are indicated by underlining. $k$ is the degree of the first power $x^{k}$ not integrated exactly by the rule.

In Tables 3-6, we give minimal CIR's with 3 and 6 points for various values of $a=\frac{1}{2}(\sqrt{ } \rho+1 / \sqrt{ } \rho)$. These rules show the asymptotic behavior both for $\rho \rightarrow \infty$ and for $\rho \rightarrow 1$. In Tables 3 and 4 are given minimal CIR's in $L^{2}\left(\varepsilon_{\rho}\right)$ and $H^{2}\left(\varepsilon_{\rho}\right)$ respectively, while in Tables 5 and 6 are given corresponding minimal CIR's subject to the constraint $w=2 / n$. Since all rules computed are symmetric, only positive abscissae are tabulated, so that the 3-point rule consists of the tabulated abscissa $x, 0$, and $-x$. All rules were computed by minimizing the norm of the error functional as a function of the abscissae and weight, where pertinent, using the method of Fletcher and Powell [4].

Remark. In Tables 3 and 6, we observe convergence of the abscissae to zero as $\rho \rightarrow 1$. We conjecture that this is true for all $n$; however, our theory is unable to prove such behavior since it takes into account only first-order terms.

Department of Applied Mathematics

Weizmann Institute of Science

Rehovot, Israel

1. R. E. BARNHILl \& J. A. WIXOM, "Quadratures with remainders of minimum norm. II," Math. Comp., v. 21, 1967, pp. 382-387. MR 36 \#6139.

2. R. E. Barnhill, J. E. DenNis, JR. \& G. M. Nielson, "A new type of Chebyshev quadrature," Math. Comp., v. 23, 1969, pp. 437-441. MR 39 \#3698.

3. P. J. DAvis, Interpolation and Approximation, Blaisdell, Waltham, Mass., 1963. MR 28 \#393.

4. R. F. FLETCHER \& M. J. D. POWELL, "A rapidly convergent descent method for minimization," Comput. J., v. 6, 1963/64, pp. 163-168. MR 27 \#2096.

5. M. Golomb \& H. F. Weinberger, Optimal Approximation and Error Bounds, Proc. Sympos. Numerical Approximation (Madison, Wis., 1958) Univ. of Wisconsin Press, Madison, Wis., 1959, pp. 117-190. MR $22 \# 12697$.

6. F. B. HildebRAND, Introduction to Numerical Analysis, McGraw-Hill, New York, 1956. MR 17, 788.

7. P. Rabinowitz \& N. Richter, "Asymptotic properties of minimal integration rules," Math. Comp., v. 24, 1970 pp. 593-609.

8. N. RiCHTER, "Properties of minimal integration rules," SIAM J. Numer. Anal., v. 6, 1969, pp. $67-79$. 